\title{
Les sources pour écrire l'histoire des cheminots dans la Résistance
}

Laurence Bour

\section{CpenEdition}

\section{Journals}

Édition électronique

URL : https://journals.openedition.org/rhcf/527

DOI : 10.4000/rhcf.527

\section{Éditeur}

Rails \& histoire

Édition imprimée

Date de publication : 1 juin 2006

Pagination : 11-25

ISSN : 0996-9403

Référence électronique

Laurence Bour, "Les sources pour écrire l'histoire des cheminots dans la Résistance », Revue d'histoire des chemins de fer [En ligne], 34 | 2006, mis en ligne le 16 mai 2011, consulté le 22 avril 2022. URL : http://journals.openedition.org/rhcf/527 ; DOI : https://doi.org/10.4000/rhcf.527 


\section{Les sources pour écrire l'histoire des cheminots dans la Résistance}

\section{Laurence Bour}

Responsable du Centre des archives historiques de la SNCF

Mener une étude historique sur la résistance cheminote, quel que soit le périmètre géographique de l'enquête, représente un long travail. Il faut en effet se livrer à une exploitation systématique de toutes les sources écrites et figurées, sans oublier la collecte de témoignages ou le recours aux archives orales qui peuvent permettre d'accéder au « dessous des choses ", aux cheminements. Au préalable, il peut être fort utile de se rapprocher des associations d'anciens résistants, par le biais notamment de la Fondation de la Résistance, afin de dresser une première liste de cheminots résistants. Coralie Immelé, auteur de travaux sur la résistance cheminote à Lyon et dans le Rhône, a également eu l'idée de recenser les noms des cheminots inscrits sur les plaques commémoratives ${ }^{1}$.

Quelles sont donc les sources à exploiter? Nous verrons en premier lieu, dans la perspective de ce colloque qui privilégie les ressources les moins connues et les plus récemment mises à la disposition de la recherche, les archives conservées par le Centre des archives historiques de la SNCF au Mans et à celui de Béziers. Nous examinerons ensuite successivement, à partir d'exemples tirés de travaux de recherche récents, les sources conservées par le Centre historique des Archives nationales à Paris, celles des Archives départementales puis des Archives municipales. Nous évoquerons enfin en quelques mots les sources détenues par l'Office national des anciens combattants et victimes de guerre et par les musées de la Résistance.

1- Coralie IMMELÉ, "La Résistance des cheminots à Lyon (1940-1944) », maîtrise d'histoire, sous la dir. de Laurent Douzou, université Lumière - Lyon II, 2001, 152 p. ; «La résistance des cheminots dans le Rhône (1940-1944) », DEA d'histoire, sous la dir. de Laurent Douzou, université Lumière - Lyon II, 2002, 157 p., avec le soutien de l'AHICF. Voir pour les plaques ce dernier mémoire, p. 69-70. 


\section{Le Centre d'archives de la SNCF à Béziers}

Ce centre assure la conservation et la communication au public des dossiers des cheminots n'ayant plus d'utilité administrative pour les services des Ressources humaines de l'entreprise ${ }^{2}$. Le centre a ouvert ses portes au public en 1998. Il conserve aujourd'hui environ $5 \mathrm{kml}$ de dossiers de carrière et/ou de dossiers de pension, et/ou de fiches carrière. Bien sûr tous les dossiers ne sont pas encore à Béziers, mais un des objectifs de la politique d'archivage de l'entreprise a bel et bien été de favoriser les transferts des dossiers d'agents sur Béziers.

Avant de se rendre à Béziers, il est préférable d'avoir dressé au préalable une liste de noms et de demander au personnel du Centre de Béziers d'effectuer des recherches à partir de ces noms. En effet, les dossiers de carrière sont fréquemment classés par année de départ de l'agent, puis seulement ensuite par ordre alphabétique. Il faut évidemment songer à la nécessité de formuler une demande de dérogation.

Un dossier de carrière se compose de cinq parties. Deux parties de ce dossier sont plus particulièrement utiles pour l'étude de la Résistance : le sous-dossier relatif aux nominations, mutations, changements de résidence, notations ; le sous-dossier des sanctions et récompenses de l'agent. Bien sûr les dossiers sont plus ou moins complets selon les cas et, finalement, assez hétérogènes. Coralie Immelé explique que, dans certains cas, elle n’y a même pas vu mentionnée l'activité résistante d'un cheminot qui était pourtant un résistant avéré ! La SNCF a pris la décision de conserver, sans les expurger, les dossiers d'agents partis à la retraite avant 1972 mais bien sûr, par le passé, des pièces ont pu être éliminées.

Malgré tout, ces dossiers sont particulièrement intéressants pour l'historien. À partir d'une large liste de cheminots morts pour la France, les dossiers d'agents permettent d'identifier de manière sûre les résistants. Nous trouvons aussi dans les dossiers des informations relatives à l'activité résistante (passages clandestins, renseignements, sabotages...) et aux organisations de résistance auxquelles sont affiliés les cheminots (Libération-Sud, NAP-Fer, Gallia, FFI).

2- Centre d'archives SNCF multirégional de Béziers, rue du Lieutenant-Pasquet, Cour Sernam, 34500 Béziers; archives.beziers@sncf.fr ; 0467496135. 


\section{Le Centre des archives historiques de la SNCF au Mans}

Depuis 2000, le Centre des archives de la SNCF a entrepris un travail de classement et d'inventaire destiné à faciliter l'accès du public aux archives de la Seconde Guerre mondiale 3 . Tous les fonds cités ont fait l'objet d'un inventaire qui vient d'être mis en ligne par l'AHICF sur le site www.trains-fr.org/ahicf, dans l'attente de leur diffusion sur le site Internet de la SNCF. Les sources ne sont pas aisées à exposer car elles sont relativement hétérogènes. Nous allons donc commencer par les plus évidentes.

\section{Les fichiers constitués par le Service central du personnel (versement 118 LM)}

Source la plus évidente et incontournable, le versement $118 \mathrm{LM}$ englobe plusieurs fichiers nominatifs de renseignements sur le personnel constitués par le Service central du personnel de la SNCF. La plupart des fichiers concerne la Seconde Guerre mondiale. Le nombre total de fiches s'élève à 89 000. Les fichiers sont uniquement consultables sur Cédéroms et après obtention d'une dérogation.

Si Paul Durand a connu et utilisé ces sources pour son étude, ce n'est en revanche pas le cas de l'historien Christian Bachelier entre 1992 et 1996. Depuis 2000, plusieurs étudiants ont eu accès à ces fichiers, mais leur sujet d'étude se limitait à la Résistance dans un secteur géographique déterminé.

Le classement d'origine des fichiers a été respecté, mais le type de classement varie d'un fichier à l'autre, ce qui en complique l'exploitation. Le dépouillement est nécessairement long. Nous allons examiner les différents types de fiches, en détaillant les renseignements qui s'y trouvent, et les thématiques abordées. Nous allons toutefois écarter les renseignements généraux qui se trouvent systématiquement sur toutes les fiches : nom et prénoms ; date de naissance ; emploi, grade et résidence administrative, adresse personnelle ; situation familiale.

Le premier type de fiche aborde deux thématiques: celle des " citations et décorations au titre de la Libération », et celle des " décorations de la médaille Résistance Fer ». Les renseignements spécifiques figurant sur ces 545 fiches sont : la date et la nature de la décoration ou de la citation; puis le texte de la citation.

3- Centre des archives historiques de la SNCF, 2, avenue de Bretagne, 72100 Le Mans ; archiveshistoriques.sncf@sncf.fr; 0243217465. 
Pour le second type de fiche, la thématique abordée est celle des « agents cités à l'ordre de la SNCF et à l'ordre de la région ». Les renseignements spécifiques sont la date et le texte de la citation. La citation inscrite sur la fiche de Léon Bronchart (118 LM34) évoque notamment son « refus de conduire un train dans lequel étaient incorporées trois voitures de détenus politiques ». Le cachet « guerre » figure sur les fiches des agents cités pour des actions liées à la Seconde Guerre. Il faut parcourir l'ensemble des 15343 fiches du fichier pour débusquer les actions de résistance car nombre de citations sont liées en fait à des actes de dévouement, de courage, ou à des blessures par faits de guerre.

2495 fiches abordent la thématique des « récompenses au titre de la Libération ». Nous y trouvons les renseignements suivants : la date et la nature de la récompense ; le texte de la citation ; le nom de l'organisme à l'origine de la proposition. Les cas de refus apparaissent dans le fichier.

Sur la thématique des «agents arrêtés, incarcérés, déportés par les Allemands pour différents motifs (vols, résistance, actes anti-allemands, et autres) » et des sous-thèmes « agents libérés, rentrés, rapatriés, décédés, disparus, fusillés », il existe 7605 fiches exploitables. Les renseignements spécifiques de ces fiches sont : la date et motif de l'arrestation; la nature de la condamnation ; la date et nom du tribunal ; la date de départ en Allemagne; le lieu d'internement; des observations soit sur la libération, la disparition, ou le décès. Toutefois, les motifs d'arrestation sont loin d'être toujours indiqués !

Un autre type de fichier couvre la thématique des « agents suspectés de communisme ou de menées antinationales, incarcérés et sanctionnés par mesure disciplinaire ", cette thématique se découpant en nombreux sous-thèmes. Le fichier compte 4619 fiches. Les renseignements spécifiques de ces fiches sont : les dates de la radiation de l'affectation spéciale, ou de l'internement, ou de l'incarcération ou encore des différentes sanctions (la suspension, le licenciement, la révocation); les appréciations de supérieurs sur l'agent (appréciations parfois absentes) ; et la mention de la correspondance échangée (celle-ci occupe parfois le recto et le verso de la fiche !).

\section{Les dossiers individuels d'agents}

Si les dossiers de carrière sont conservés à Béziers, en revanche le Centre des archives historiques de la SNCF conserve au Mans des dossiers individuels administratifs tenus par les services du personnel à 
tous les échelons de l'entreprise, du central au local. Ces dossiers constituent donc bien des sources complémentaires, même si les lacunes se déduisent elles-mêmes de l'exposé qui suit. La consultation de ces dossiers est soumise à dérogation.

Dans le fonds du Service central du personnel, nous trouvons : des dossiers d'agents arrêtés par les Allemands pour menées antinationales, essentiellement des agents communistes (cote 25 LM259) ; des dossiers de révisions de carrières d'agents membres du réseau Résistance-Fer (cotes 25 LM252 et 253); des dossiers de secours pour les réfractaires au STO non affiliés à un organisme de résistance (cote 25 LM265/3).

Dans le fonds de la Région ouest, Service Exploitation, nous trouvons les dossiers individuels d'agents - uniquement des agents de l'Exploitation donc - sanctionnés pour activité antinationale (cotes 201 LM1, 2, 3 et 4).

Dans le fonds de la Région nord sont conservés, sous les cotes 279 LM1 à 13, les dossiers d'agents de la Région nord arrêtés et incarcérés par les autorités d'occupation pour différents motifs (vols, actes anti-allemands, acte de résistance, motifs inconnus, etc.).

Dans le fonds de la Région sud-ouest, Service Matériel et Traction, sont conservés sous les cotes 303 LM11, 12, 13, 14, 15 et 73 LM29, 30, les dossiers individuels d'agents arrêtés et incarcérés par les autorités allemandes pour divers motifs : filiation juive, faits d'ordre politique (activité communiste, tendance gaulliste) ; franchissement de la ligne de démarcation sans laissez-passer ; transport illicite de correspondance en zone libre; vols; réflexions injurieuses à l'égard des Allemands; prise de photographies.

Coralie Immelé a pu exploiter quelques dossiers présents dans les archives de la Région SNCF du Sud-Est.

Beaucoup de ces dossiers administratifs relatifs aux agents arrêtés et incarcérés sont liés aux questions de versement de la solde, des allocations familiales, ou de secours. Mais ils comportent aussi des renseignements relatifs aux faits reprochés à l'agent, à la sanction prise, à l'arrestation. Leur typologie documentaire est très riche puisqu'ils contiennent de la correspondance, divers imprimés de renseignements, divers formulaires, des rapports préfectoraux, etc.

4- Coralie IMMELÉ, «La résistance des cheminots dans le Rhône (1940-1944) », DEA cité, p. 59-60 
Il ne faudrait pas négliger les dossiers relatifs aux mesures disciplinaires prises pendant la Seconde Guerre par le conseil de discipline présents dans les archives du Service Matériel et Traction de la Région sud-ouest sous les cotes 303 LM6, 7, 8, 9. On y trouve les séances du conseil de discipline pendant la guerre, puis des dossiers individuels d'agents sanctionnés par des mesures disciplinaires.

\section{Approche thématique, non exhaustive}

Notre première thématique aborde les éléments contextuels. Toute étude historique sur la résistance cheminote débute en plantant le « décor », c'est-à-dire en présentant les relations avec les autorités allemandes, l'organisation des services allemands dans les territoires occupés de la SNCF, et les contraintes qui en résultent. Sans oublier la présentation de la corporation cheminote, etc. Sur ce point, les archives de l'entreprise sont primordiales, comme le montrent le fonds Durand évoqué ci-dessous et les « documents SNCF » qui le composent. Nous n'allons pas nous étendre ici et maintenant sur le sujet, mais nous renvoyons le chercheur à l'État sommaire des archives de la période 19381948 qui figure sur le site Internet de l'AHICF. Les archives clés permettant d'étudier les relations avec l'occupant y sont exposées : archives du Secrétariat du conseil d'administration, archives du Secrétariat de la direction générale, archives du Service technique de la direction générale. Il ne faut pas négliger les archives de la Région nord (PNV 3915, PNV 3918, PNV 3920) : les dossiers relatifs à la surveillance des voies sont fort intéressants.

La thématique que nous souhaitons ensuite aborder est celle des formes d'actions de résistance.

Ces formes d'action s'analysent au travers de la lutte que mène l'entreprise à leur encontre, notamment par le biais des affiches : lutte contre les sabotages, contre les indiscrétions professionnelles de nature à renseigner l'ennemi, contre les vols, contre les transports de correspondance entre la zone libre et la zone occupée. Cette lutte transparait également au travers des textes réglementaires de l'entreprise. Nous songeons en premier lieu aux ordres du jour destinés à l'ensemble du personnel, tel l'Ordre du jour 47 du 20 octobre 1942, signé Le Besnerais, directeur général, sur le secret des transports, ou encore l'Ordre du jour 51 du 28 avril 1943, signé Bichelonne, ministre à la Production industrielle et aux Communications, lançant un appel contre les attentats. 
Mais il ne faut pas négliger l'ensemble des autres textes réglementaires, nationaux et locaux, tel l'Avis général $\mathrm{n}^{\circ} 8$ relatif à la correction à observer à l'égard des autorités d'occupation datant de janvier 1941. Les collections du Centre des archives de la SNCF au Mans, même si elles souffrent encore de lacunes, surtout pour les règlements émanant des directions régionales et des établissements, sont aujourd'hui inventoriées.

Parmi les formes d'actions figurent les tracts. Le Centre des archives historiques de la SNCF au Mans détient, dans le fonds du Service central du personnel, une quantité importante de tracts sous les cotes 25 LM258 et 25 LM1934, dont un échantillon raisonné est publié dans le présent volume.

Quand aux vols, ils sont une des formes possibles d'actions de résistance lorsqu'ils sont orientés contre les autorités occupantes. La cote 25 LM240 contient des archives du Service central du personnel relatives aux vols commis à la SNCF pendant la période de guerre : statistiques, procès-verbaux des commissions de surveillance générale ; affichage des mesures disciplinaires.

Une autre forme essentielle d'actions de résistance réside dans les actes de sabotage. Le Centre du Mans conserve un certain nombre d'archives relatives aux faits de guerre, terme qui recouvre les bombardements, mitraillages et les sabotages. Mais l'apport de ces archives pour l'étude de la résistance cheminote est loin d'être évident, car il faut ensuite pouvoir lier les sabotages à l'action de cheminots! Ainsi, il semble qu'il faille écarter les archives, assez nombreuses, relatives aux cheminots blessés et/ou tués, et sinistrés par faits de guerre. Nous déplorons, au niveau des archives des services centraux, d'importantes lacunes dans les archives du Service du Mouvement, lacunes partiellement comblées par le fonds Durand. Du côté des archives des services régionaux, le fonds du Service Voie et Bâtiments de la région de l'Est est de loin le plus intéressant, puisqu'il est permet de dresser la cartographie des dégâts liées aux faits de guerre. Le versement concerné est 111 LM. Nous y trouvons : les rapports sur les sabotages et cela pour tous les arrondissements de la Région est; la correspondance avec le Service central du personnel relative aux incidents et dégâts causé au chemin de fer par des actes de sabotage; les dépêches du poste régional d'information concernant les incidents de circulation des trains dus aux bombardements, sabotages et mitraillages. 
Sur les évasions, citons la cote 25 LM1940/4 qui fournit la liste des agents ayant facilité le passage de la ligne de démarcation ou ayant facilité l'évasion de prisonniers en France, en Allemagne ou en Autriche.

Au sujet de l'appartenance à des organisations (groupes, réseaux, mouvements, mouvance communiste, NAP-Fer), citons la cote 25 LM1940/4 où figurent les listes nominatives d'agents arrêtés par les Allemands et décédés en France ou en déportation.

Avant de conclure, nous ne pouvons omettre, dans ce panorama général des sources sur la résistance cheminote, la série de cassettes vidéo enregistrées en lien avec le $8^{e}$ colloque de l'AHICF de juin 2000. Le chercheur dispose là de vingt témoignages de résistants, dont notamment deux résistants de Limoges et deux de Lyon. Ces enregistrements, dont la direction de la Communication de la SNCF était le commanditaire, ont été produits par le Centre audiovisuel de la SNCF et peuvent être consultés au Mans et à l'AHICF à Paris.

Voyons à présent les sources offertes par les autres fonds d'archives publics et privés, telles qu'elles ont été utilisées par des travaux récents.

\section{Sources conservées au Centre historique des Archives nationales (CHAN), à Paris}

\section{AJ : Fonds du Comité d'histoire de la Deuxième Guerre mondiale}

Le fonds le plus intéressant est celui du Comité d'histoire de la Deuxième Guerre mondiale. Il est coté $72 \mathrm{AJ}$, sachant que la série AJ accueille les fonds divers remis aux Archives nationales.

Lors du $8^{e}$ colloque de l'Association pour l'histoire des chemins de fer en France, "Une entreprise publique dans la guerre : la SNCF, 1939-1945 », les 21 et 22 juin 2000, Patricia Gillet a présenté cette série 72 AJ comme une "stratification empirique de pièces originales, de documents de travail, de témoignages et de papiers personnels $»^{5}$. En effet, elle regroupe les documents collectés, entre 1951 et 1980, par les membres de cet organisme, chargés de susciter des témoignages sur la Résistance, sur l'occupation, et de réaliser des enquêtes et des publications sur la période de guerre. La série accueille aussi les archives personnelles

5- Patricia GILLET, «L'apport des archives du Comité d'histoire de la Deuxième Guerre mondiale à l'histoire de la $\mathrm{SNCF}$ ", in $\mathrm{AHICF}$, Une entreprise publique dans la guerre. La SNCF, 1939-1945, Paris, PUF, 2001, p. 45-50, voir p. 49. 
des membres du comité. Au-delà de 1980, année de sa dissolution, la série s'est enrichie d'archives privées sur la Seconde Guerre.

Au sein de la série $72 \mathrm{AJ}$, la place d'honneur revient au fonds Paul Durand coté 72 AJ 473 à 508

Ce fonds de trente-six cartons comprend la documentation rassemblée par Paul Durand, inspecteur général honoraire de la SNCF, en vue de son ouvrage La SNCF pendant la guerre paru en 1968. L'inventaire du fonds est en ligne sur le site consacré par l'AHICF à l'actualité de la recherche historique sur les chemins de fer français et les cheminots pendant la Deuxième Guerre mondiale (www.ahicf.com).

En tant qu'ancien cheminot, Paul Durand a largement recouru aux archives de l'entreprise. Il a été personnellement mandaté par le président Louis Armand pour s'approprier quantité de pièces ou du moins en prendre copie. Nous trouvons donc dans le fonds Durand le fruit de ses travaux de collecte de «documents SNCF ». Ces archives permettent d'étudier les conditions d'exploitation du réseau et les relations entretenues avec les autorités d'occupation.

Outre le corpus de «documents SNCF», le fonds Paul Durand doit sa richesse aux archives relatives à l'enquête qu'il a menée sur le rôle des cheminots pendant la guerre (cotes 72 AJ 495 à 498). En introduction de son ouvrage, Paul Durand évoque l'envoi de « 417 questionnaires individuels adressés à des résistants indiscutables. 114 seulement ont été retournés ».

\section{Après le fonds Paul Durand, signalons les cotes 72 AJ 74-75}

Ce fonds est improprement appelé «Résistance Fer (SNCF)》. En fait, les documents qui le composent sont un « condensé » de l'enquête de Paul Durand. Ils décrivent essentiellement les actes de sabotage commis par les cheminots ainsi que les résultats obtenus, mais aussi les dégâts causés par les bombardements alliés.

\section{Les témoignages et documents recueillis après guerre par les correspondants locaux du Comité d'histoire}

Ils sont classés par département, sous les cotes 72 AJ 90-209, ou par mouvement et réseau de résistance sous les cotes 72 AJ 35-81. En tête de ces dossiers, nous trouvons les relevés des faits de résistance dans des documents allemands, ou les relevés des faits de résistance tirés du journal FTPF France d'abord. 


\section{Parmi les fonds privés ajoutés à la série 72 AJ au-delà de 1980,} figure le fonds de l'association Résistance-Fer coté 72 AJ 2280-2297

L'association Résistance-Fer a déposé ses archives au Centre historique des Archives nationales au moment de sa dissolution, en mai 2000. Le répertoire numérique détaillé datant de septembre 2001, le fonds n'est accessible aux chercheurs que depuis peu de temps. L'inventaire du fonds est en ligne sur le site www.ahicf.com. Coralie Immelé, en se limitant volontairement, dans le cadre restreint de son DEA, à l'étude des documents de la période 1940-1944, a exploité avec profit trois cotes sur les dix-huit car la plupart des papiers concerne la période postérieure 6 .

Nous trouvons dans ce fonds :

- des documents sur la fondation et l'évolution de l'association (72 AJ 2280);

- des rapports des organes centraux (dont les procès-verbaux des réunions hebdomadaires du Bureau d'action immédiate à partir d'octobre 1944), congrès et assemblées générales de l'association ;

- des archives relatives aux homologations des membres de Résistance-Fer, dont des états nominatifs, un registre des homologations (72 AJ 2286) ;

- des archives sur l'aide sociale apportée aux familles des résistants disparus, fusillés ou morts en déportation, dont des fiches nominatives ;

- des archives relatives à la défense de la mémoire de la Résistance et de la Déportation ;

- des documents sur la participation aux activités de fédérations et d'associations liées à la résistance ferroviaire ;

- des documents relatifs aux manifestations et cérémonies commémoratives ;

- des publications de l'association.

Nous conclurons la présentation de la série $72 \mathrm{AJ}$ en rappelant à nouveau les propos de Patricia Gillet au colloque de juin 2000 : «C'est du côté de la Résistance que se situent les gisements les plus importants » de la série $72 \mathrm{AJ}^{7}$.

6- Coralie IMMELÉ, «La résistance des cheminots dans le Rhône (1940-1944) », DEA cité, p. 45.

7- Patricia GILLET, «L'apport des archives du Comité d'histoire de la Deuxième Guerre... », art. cité, p. 49. 


\section{Fonds 3 AG 2, archives du « Bureau central de renseignements et d'action (BCRA) "}

Outre la série $72 \mathrm{AJ}$, il nous faut mentionner une source d'un grand intérêt présente au Centre historique des Archives nationales. Il s'agit du fonds du Bureau central de renseignements et d'action (BCRA), coté 3 AG 2, sachant que la série 3 AG correspond aux archives de Gaulle antérieures à 1959. Le BCRA était chargé d'assurer la liaison entre la Résistance métropolitaine d'une part, la France libre et les Alliés d'autre part. Les cotes relatives à la SNCF sont les suivantes : 3 AG 2 234, 235, 236, 361.

\section{Série $F$ 7, archives des Renseignements généraux et des services de police}

Des éléments peuvent être trouvés au sein de la série F 7 dans les archives des Renseignements généraux et des services de police.

Les fonds des Renseignements généraux incluent de la documentation sur la SNCF, des dossiers sur les sabotages et les attentats permettant de reconstituer l'histoire de la Résistance.

La série F 7 comprend aussi :

- les dossiers d'organisation et de fonctionnement du corps des gardes de communication, chargés de surveiller les voies et les ateliers de la SNCF ;

- les registres de main courante du commissariat de la gare du Nord.

\section{Sources conservées aux Archives départementales (AD)}

Nous allons maintenant présenter les sources conservées aux Archives départementales, mais bien sûr ces sources varient d'un département à l'autre. Le chercheur doit bien évidemment consulter les inventaires disponibles. Lors des recherches dans les inventaires, il ne faut pas négliger les sources orales.

\section{Archives des services préfectoraux}

Examinons en premier lieu les archives des services préfectoraux : archives du cabinet du préfet et archives des services de police et de gendarmerie.

Les rapports des préfets sont des sources très utiles car ils sont rédigés à partir de rapports très détaillés des services administratifs et 
des sous-préfets. Ils traitent de l'état d'esprit de la population, des actes de sabotages, de l'état du trafic, des relations avec les autorités allemandes.

Au sein des archives préfectorales, les sources les plus fréquemment rencontrées sont les documents relatifs aux destructions causées par les actes de résistance et par les bombardements. Dans les départements au trafic ferroviaire de forte importance ou d'un fort niveau d'activité de résistance, la proportion de dossiers concernant les sabotages ferroviaires peut être élevée. Ces dossiers constituent des sources précises et diverses du fait de la présence de multiples rapports : rapport du sous-préfet au préfet, rapport de gendarmerie, rapport du préfet à la direction générale de la Police à Vichy, rapport des Renseignements généraux. Par ailleurs, les archives témoignent de l'étroite surveillance dont faisaient l'objet les cheminots, par crainte de propagande communiste.

\section{Fonds judiciaires}

Examinons maintenant les fonds judiciaires : archives des tribunaux et archives des établissements pénitentiaires.

Archives des tribunaux

Coralie Immelé a ainsi entrepris une étude des archives des juridictions d'exception instaurées par le gouvernement de Vichy à Lyon : sections spéciales et tribunal spécial, tribunal d'État. Les dossiers de justice constituent bien une source complémentaire permettant de mieux connaitre la vie clandestine des résistants. Attention toutefois, ces archives ne sont consultables qu'après obtention d'une dérogation ${ }^{8}$.

\section{Archives des établissements pénitentiaires}

Prenons à nouveau l'exemple du travail de Coralie Immelé. Elle a exploité les archives de la prison militaire de Montluc en utilisant le fichier des fusillés, disparus, déportés, libérés, rapatriés afin d'identifier les cheminots résistants emprisonnés, puis en consultant leurs dossiers, ces derniers étant toutefois de composition assez inégale?

\section{Archives de la série J}

Les fonds présents dans la série J, qui accueille les fonds entrés par voie extraordinaire, peuvent varier d'un département à l'autre, en fonction des dépôts d'archives effectués. Par exemple, les Archives

\footnotetext{
8- Ibid., p. 51-52.

9- Ibid., p. 53-54.
} 
départementales de l'Aisne conservent des fichiers chronologiques des bombardements et mitraillages, ainsi que des listes de sabotages par réseaux de résistance.

\section{Archives (locales) du Comité d'histoire de la Deuxième Guerre mondiale}

Des archives se retrouvent systématiquement dans toutes les Archives départementales, il s'agit des archives des correspondants locaux du Comité d'histoire de la Deuxième Guerre mondiale. Ils ont déposé aux Archives départementales le double de leur travail et leurs documents préparatoires.

\section{Par ailleurs il ne faut pas ignorer les périodiques conservés par les archives départementales}

Ainsi, Coralie Immelé a exploité, aux AD du Rhône, Le Rail, organe corporatif ferroviaire. Ce bimensuel, qui s'adressait uniquement aux cheminots, était un journal proche du pouvoir. Il communique les mesures adoptées par l'entreprise. On y voit de manière indirecte certaines formes de résistance utilisées par les cheminots : transports clandestins de courriers, sabotages.

\section{Aux Archives municipales (AM)}

Les données relatives aux sources conservées par les Archives municipales sont encore plus incertaines.

Par supposition, nous pouvons penser que les communes ayant été des nœuds ferroviaires ou ayant hébergé des centres et des installations ferroviaires doivent conserver des archives plus conséquentes que les autres communes. Toutefois Mélanie Gouiffès n'a trouvé à Bordeaux que des périodiques locaux ${ }^{10}$ et Coralie Immelé a «seulement trouvé [aux AM de Lyon] des photographies montrant les dégâts causés par les bombardements alliés dans les enceintes ferroviaires $»^{11}$.

Nous sortons maintenant des « sentiers classiques » des archives nationales, départementales et municipales pour nous tourner vers les archives de quelques autres organismes. Évoquons en premier lieu l'ONAC.

10- Mélanie GOUIFFÈS, «La résistance des cheminots en Gironde durant le Second conflit mondial ", maitrise d'histoire, sous la dir. de Ch. Bouneau, université Michelde-Montaigne - Bordeaux III, 2001, 211 p., avec le soutien de l'AHICF. Voir p. 196. 11- Coralie IMMELÉ, «La résistance des cheminots dans le Rhône (1940-1944)», DEA cité, p. 56. 


\section{Office national des anciens combattants et victimes de guerre (ONAC)}

Cet établissement public, créé en 1916 pour soutenir les mutilés et réformés de la Première Guerre mondiale, a pour mission première de prendre en charge le droit à réparation reconnu par la loi aux anciens combattants et victimes de guerre. Les services départementaux de l'ONAC sont présents dans tous les départements de France. Le chercheur doit donc solliciter le service ou les services départementaux souhaités. Toutefois les cheminots pour qui a été déposé un dossier à l'ONAC sont les cheminots déclarés « Morts pour la France » : ils ont été fusillés, ou sont morts en déportation, ou décédés lors de bombardements, mais ils n'ont pas forcément été des résistants.

Le questionnaire du dossier comprend des renseignements concernant l'état civil, la participation à un groupe de résistance, les actions, les déportations ; des témoignages de résistants doivent étayer le dossier.

\section{Archives syndicales}

Avec les archives syndicales nous entrons dans le domaine des archives privées. Les deux centrales dominantes au sein du monde cheminot entre 1940 et 1944 étaient la CGT (Confédération générale des travailleurs) et la CFTC (Confédération française des travailleurs chrétiens). Mais on a pu recenser 733 syndicats locaux en 1938 et encore 379 en décembre 1943.

\section{Institut CGT d'histoire sociale de la Fédération des cheminots de Montreuil}

Madeleine Peytavin évoquait, au colloque de juin 2000, la décision de l'Institut d'histoire sociale de la Fédération des cheminots de Montreuil de déposer en plusieurs étapes, et après classement, ses archives aux Archives départementales de la Seine-Saint-Denis. En fait, le classement de l'ensemble des archives détenues par l'institut est encore en cours.

\section{CFTC}

Les archives de la CFTC de la période de la Seconde Guerre sont détenues par le Service des archives confédérales de la CFDT créé en avril 1985. 
Les sources les plus évidentes au niveau des archives syndicales sont les publications clandestines : La Tribune des cheminots pour la CGT ; et Le Cheminot de France CFTC. Mais la composition de ces fonds et leur intérêt du point de vue de la recherche sur la résistance cheminote restent à approfondir. Coralie Immelé, quant à elle, n'a pu accéder aux archives de la CFTC dans le cadre de son mémoire de DEA ${ }^{12}$.

\section{Nous allons maintenant évoquer quelques musées et centres d'histoire de la Résistance}

Le centre de documentation du Centre d'histoire de la Résistance et de la Déportation (CHRD), à Lyon, conserve un fonds spécialisé. Par ailleurs son fonds d'archives est constitué en majorité d'archives privées d'anciens résistants et/ou de déportés, parmi lesquels se trouvent des cheminots résistants : Albert Chifflot, Nambotin, Peytier, Verlhac comme l'a établi Coralie Immelé ${ }^{13}$.

Le Musée de la Résistance nationale de Champigny-sur-Marne détient une importante collection de tracts et de périodiques clandestins.

Le Musée de l'histoire vivante à Montreuil conserve lui des témoignages de résistants.

Avec la disparition progressive des témoins de l'époque, le chercheur devra de plus en plus recourir aux sources archivistiques. L'extrême dispersion de ces sources impose une grande mobilité aux chercheurs, d'autant que de nouveaux gisements encore inconnus vont nécessairement être mis au jour, y compris à la SNCF, nous l'espérons. Chacun des lieux de conservation mentionnés dans cet exposé détient des documents permettant de nourrir l'étude historique, mais chacun des lieux présente aussi des lacunes. Il appartient à l'historien de confronter les sources et de les exploiter de façon complémentaire.

12- Ibid., p. 66.

13- Ibid., p. 67-69. 
